Cahiers de la recherche sur les droits

Cahiers

Fon les Droits fondamentaux

3 | 2004

Surveiller et punir / Surveiller ou punir ?

\title{
Homosexualité et droit de la famille
}

\section{Sylvaine Cheval}

\section{OpenEdition}

Journals

Édition électronique

URL : https://journals.openedition.org/crdf/7546

DOI : $10.4000 /$ crdf. 7546

ISSN : 2264-1246

Éditeur

Presses universitaires de Caen

Édition imprimée

Date de publication : 1 octobre 2004

Pagination : 93-102

ISBN : 2-84133-221-7

ISSN : $1634-8842$

Référence électronique

Sylvaine Cheval, «Homosexualité et droit de la famille», Cahiers de la recherche sur les droits fondamentaux [En ligne], 3 | 2004, mis en ligne le 18 décembre 2020, consulté le 14 novembre 2022 URL : http://journals.openedition.org/crdf/7546 ; DOI : https://doi.org/10.4000/crdf.7546 


\title{
Homosexualité et droit de la famille ${ }^{1}$
}

\author{
Sylvaine CHEVAL \\ Doctorante à l'Université du Havre
}

I. Des institutions familiales réfractaires à l'homosexualité? [Étude de droit positif]

A. Le lien matrimonial

B. La filiation

II. Une évolution inéluctable ? [Étude de droit prospectif]

A. L'existence de pressions extérieures

B. La nécessité de contrôler l'évolution

Le terme «homosexualité», «néologisme barbare jailli d'un mélange monstrueux de racines grecques et latines $»^{2}$, n’a été forgé qu'au XIX siècle par la médecine qui voyait dans la préférence sexuelle pour une personne de même sexe un comportement déviant ${ }^{3}$. Auparavant, sous l'influence de la religion, l'homosexualité était déjà l'objet d'une réprobation sociale parfois virulente. Elle était ainsi passible de mort dans tout l'Occident chrétien jusqu'à la fin du XVIII siècle et, en France, l'époque n'est pas si lointaine où les homosexuels pouvaient être livrés au bûcher ${ }^{4}$. Si de telles abominations n'ont plus cours, on peut toutefois rappeler qu'en 1960 l'amendement Mirguet classait l'homosexualité parmi les fléaux sociaux contre lesquels il était nécessaire de lutter, au même titre que la prostitution, l'alcoolisme ou la tuberculose et qu'elle a été considérée jusqu'en 1981 comme une maladie mentale ${ }^{5}$. Il est également significatif de mentionner que ce n'est qu'en 1982 qu’a été abrogé le dernier texte incriminant spécifiquement les actes homosexuels ${ }^{6}$.

Aujourd'hui, les homosexuels semblent mieux acceptés par l'opinion publique et le droit, après les avoir longtemps opprimés, a consenti à les placer sous sa protection. À l'heure actuelle, toute discrimination fondée sur l'orientation sexuelle est ainsi prohibée. L'article 14 de la Convention européenne des droits de l'homme a été interprété en ce sens ${ }^{7}$ et la législation interne a également affirmé ce principe dans différents textes ${ }^{8}$. Parallèlement, la jurisprudence a eu l'occasion de reconnaitre des droits aux

1. Ce texte est issu d'une communication présentée lors d'un colloque organisé par l'Université du Havre, à l'occasion du bicentenaire du Code civil, sur le thème «La famille dans tous ses états».

2. H. Ellis, L'Inversion sexuelle, 1897.

3. K.M. Benkert, dit Kertbeny, Das Gemeinschädliche des \$143 des prussischen Strafgsetzbuches, 1869 (premier usage avéré du terme « Homosexualität»).

4. En 1783, un religieux qui avait commis un acte sexuel avec un homme a été brûlé vif. Il s'agit de la dernière condamnation à mort pour ce motif exécutée en France.

5. F. Martel, Le Rose et le Noir. Les homosexuels en France depuis 1968, Paris, Seuil, 2000.

6. Étaient alors réprimés les actes homosexuels consentis entre un majeur et un mineur âgé de moins de dix-huit ans (art. 331-2 C. pén.). Il convient toutefois de rappeler que le Code pénal adopté par l'Assemblée constituante révolutionnaire en 1791, puis le Code Napoléon de 1810 (sous l'influence de Cambacérès) avaient exclu toute condamnation spécifique de l'homosexualité et que ce n'est qu'en 1942 qu'une législation répressive a été réintroduite par le gouvernement de Vichy. L'article 331-2 du Code pénal était l'un des vestiges de ces lois vichystes.

7. «La jouissance des droits et libertés reconnus dans la [...] Convention doit être assurée, sans distinction aucune, fondée notamment sur le sexe, la race, la couleur, la langue, la religion, les opinions politiques ou toutes autres opinions, l'origine nationale ou sociale, l'appartenance à une minorité nationale, la fortune, la naissance ou toute autre situation ».

8. On peut notamment citer l'article 225-1 du Code pénal ou l'article L 122-45 du Code du travail. 
homosexuels ${ }^{9}$ et une loi visant à réprimer l'homophobie est actuellement à l'étude. Il reste néanmoins aux homosexuels un dernier bastion à conquérir: le droit de la famille. Cette branche du droit, qui a vocation à régir les relations entre parents et alliés, semble de prime abord peu disposée à concéder une place aux homosexuels. Il convient dès lors d'examiner si elle est susceptible de s'adapter à l'évolution des mœurs et des mentalités.

Le couple homosexuel a d'ores et déjà accédé à une certaine reconnaissance juridique ${ }^{10}$, comme en témoigne la nouvelle définition du concubinage issue de la loi du 15 novembre 1999 qui, à l'encontre d'une jurisprudence constante de la Cour de cassation ${ }^{11}$, y inclut les relations entre deux personnes de même sexe ${ }^{12}$. Cette loi a également instauré le Pacte civil de solidarité (ou PACS), contrat qui permet à deux personnes physiques « d'organiser leur vie commune ${ }^{13}$. Cette nouvelle forme de conjugalité s'est voulue accessible aux hétérosexuels comme aux homosexuels. En revanche, les institutions caractéristiques du droit de la famille que sont le mariage et la filiation paraissent peu compatibles avec l'homosexualité. Cependant, la société évolue, la Cour européenne des droits de l'homme développe une approche plus éclectique de la famille ${ }^{14}$ et l'on peut se demander si, bien que les institutions familiales françaises semblent effectivement souvent hostiles à l'homosexualité (I), une évolution ne se profile pas inéluctablement (II).

\section{Des institutions familiales réfractaires à I'homosexualité ? [Étude de droit positif]}

Le mariage est traditionnellement conçu comme l'union d'un homme et d'une femme. La France, dans toute son histoire, n'a connu l'union matrimoniale que sous sa forme hétérosexuelle: les homosexuels en sont donc exclus. Similairement, dès lors que deux personnes de même sexe ne peuvent procréer ensemble, la filiation apparaît totalement étrangère à l'homosexualité. L'affaire est-elle définitivement entendue? En réalité on peut constater, en dépit de ces certitudes, que l'homosexualité n'est pas absente des questions relatives au lien matrimonial (A) ni de celles concernant la filiation (B).

\section{A. Le lien matrimonial}

L'homosexualité d'un conjoint peut tout d'abord justifier la rupture du mariage. Elle est ainsi susceptible de constituer une cause de nullité du mariage, l'erreur sur l'orientation sexuelle de l'époux pouvant être analysée comme une erreur sur une qualité essentielle de la personne ${ }^{15}$. Toutefois, l'homosexualité apparaît surtout dans le contentieux du divorce pour faute ${ }^{16}$. L'entretien par l'un des époux d'une relation homosexuelle constitue à cet égard une violation grave ou renouvelée des devoirs du mariage. Il s'agit en effet d'un manquement à l'obligation de fidélité édictée par l'article 212 du Code civil, manquement que l'on peut qualifier d'adultère ${ }^{17}$, bien que les juridictions saisies semblent réticentes à employer ce terme. Le qualificatif retenu est alors celui de comportement injurieux envers le conjoint ${ }^{18}$. En tout état de cause, l'étude de la jurisprudence montre que le grief d'homosexualité, lorsqu'il est établi, entraîne systématiquement le prononcé du divorce ${ }^{19}$.

Est davantage problématique la question du mariage de deux personnes de même sexe. Peut-on considérer le mariage comme irréductiblement hétérosexuel ? La jurisprudence n'a jamais eu d'hésitation sur ce point ${ }^{20}$ et le

9. Une femme a ainsi pu se voir indemniser du préjudice par ricochet que la mort accidentelle de sa compagne lui avait occasionné (TGI Belfort, 25 juillet 1995, PA, 2 mars 1998, p. 13, note I. Corpart, JCP Ed. G., 1996, II 22724, note C. Paulin). Le tribunal a d'ailleurs calculé le montant de l'indemnisation requise en se fondant sur les principes « dictant la détermination du préjudice d'un conjoint survivant».

10. La reconnaissance des couples de même sexe implique déjà l'intégration des homosexuels dans le droit de la famille, tant il est vrai qu'un couple constitue une structure de type familial. Les juges anglais ont ainsi considéré qu'un concubin homosexuel était pour son compagnon un membre de la famille (J. Flauss-Diem, "Couple de même sexe et famille, version anglaise (à propos de la décision Fitzpatrick du 28 octobre 1999) ", Dr fam., $\mathrm{n}^{\circ} 24$, 2000). Il est également significatif que les ouvrages consacrés au droit de la famille traitent tous du concubinage et du PACS.

11. Cass. civ. $3^{\mathrm{e}}, 17$ décembre 1997 (D., 1998, p. 111, concl. J.-F. Weber et note J.-L. Aubert, JCP Ed. G., 1998, II 10 093, note A. Djigo) : « Le concubinage ne [peut] résulter que d'une relation stable et continue ayant l'apparence du mariage, donc entre un homme et une femme.» Cette affirmation pouvait toutefois être contestée. L'étymologie du terme « concubinage " (du latin «concubina» : qui couche avec) n'impliquait aucunement d'exclure les couples de même sexe et, de surcroît, il était possible de soutenir qu'exiger une différence de sexe pour que la relation entre deux personnes soit qualifiée de concubinage revenait à ajouter à la loi une condition qu'elle ne postulait nullement.

12. Art. 515-8 C. civ., issu de la loi $\mathrm{n}^{\circ}$ 99-944 du 15 novembre 1999: «Le concubinage est une union de fait, caractérisée par une vie commune présentant un caractère de stabilité et de continuité, entre deux personnes de sexe différent ou de même sexe, qui vivent en couple. »

13. Art. 515-1 C. civ.

14. En reconnaissant notamment aux transsexuels le droit de se marier (arrêts Christine Goodwin c. Royaume-Uni, req. $n^{\circ} 28957$ / 95 et I. c. Royaume-Uni, req. $\mathrm{n}^{\circ} 25680 / 94$, rendus le 3 juillet 2002).

15. Au sens de l'article 180 alinéa 2 du Code civil. Ce vice du consentement entacherait alors le mariage d'une nullité relative.

16. A. Gouron-Mazel, «Juge de la famille et homosexualité», Dr fam., $\mathrm{n}^{\circ} 1,2002$, p. 4.

17. On peut souligner ici que ce n'est pas l'homosexualité en elle-même qui est fautive, mais le manquement à l'obligation de fidélité qu'elle implique.

18. Les deux qualificatifs sont en fait envisageables pour désigner l'entretien par un époux d'une relation homosexuelle. Pourrait également être envisagé le manquement à l'obligation de loyauté dégagée par la jurisprudence (P. Courbe, Droit de la famille, $2^{\mathrm{e}}$ éd., Paris, Armand Colin, 2001, $\mathrm{n}^{\circ} 355$ ) lorsqu'il y a dissimulation d'une homosexualité connue avant le mariage.

19. On peut toutefois mentionner à titre anecdotique un arrêt de la Cour d'appel d'Aix-en-Provence du 22 avril 1999 (Juris-Data nº 1999-041698) qui a refusé de prononcer le divorce, l'épouse ayant accepté, de longues années durant, la présence au domicile conjugal de l'amant de son mari jusqu'à ce que les deux hommes quittent ensemble ce domicile. L'adultère, en l'espèce, ne rendait manifestement pas intolérable le maintien de la vie commune. En dehors de telles hypothèses marginales, il apparaît que l'homosexualité d'un époux peut lui être opposée pour rompre le mariage.

20. Civ. 6 avril 1903, DP, 1904, 1, 395, concl. Bourdoin, S., 1904, 1, 273, note Wahl. 
droit positif semble bien fixé en ce sens ${ }^{21}$. Toutefois, la lecture des dispositions du Code civil qui régissent cette institution laisse quelque peu perplexe. En effet, le Code n'exige pas expressément la différence de sexe parmi les conditions du mariage. Certes, en 1804, la question du mariage entre homosexuels était inconcevable et il n'est guère surprenant que les rédacteurs du Code civil n'aient pas perçu la nécessité d'édicter une interdiction formelle. N'est-ce pas néanmoins ajouter à la loi que d'en exclure les couples de même sexe? Ubi lex non distinguit... On peut de surcroît relever que les personnes mariées ne sont désignées que par le terme d' «époux », ce qui ne permet nullement de déduire qu'il doive s'agir d'un homme et d'une femme. Quant à l'article 144, souvent évoqué comme impliquant que les époux doivent être de sexe différent, il se borne à énoncer que "l'homme avant dix-huit ans révolus, la femme avant quinze ans révolus, ne peuvent contracter mariage ». Pris à la lettre, il pourrait être interprété comme exigeant, dans l'hypothèse d'un mariage entre deux femmes, qu'elles aient chacune plus de quinze ans, ou bien pour deux hommes qu'ils aient tous deux atteint l'âge de dix-huit ans ${ }^{22}$. Il n'y a guère que l'article 75 , relatif aux actes de mariage, qui fasse référence expresse à la différence de sexe requise des époux: il y est énoncé que l'officier d'état civil « recevra de chaque partie, l'une après l'autre, la déclaration qu'elles veulent se prendre pour mari et femme». La preuve de l'hétérosexualité du mariage semble faite. Il peut toutefois paraître surprenant qu'une disposition relative aux actes de l'état civil conditionne tout le régime d'une institution comme le mariage. Il serait aisé de passer outre, sinon à la faveur d'une interprétation prétorienne ${ }^{23}$, du moins par une réforme législative facilitée par ce vide juridique.

Le rôle du mariage dans notre société est au cœur du débat. Portalis définissait le mariage comme «la société de l'homme et de la femme qui s'unissent pour perpétuer leur espèce, s'aider, par des secours mutuels, à porter le poids de la vie et partager leur destinée commune ${ }^{24}$. Or, il est indéniable que la signification juridique du mariage a changé. Ainsi, s'il représentait jadis l'union de deux familles, il est surtout aujourd'hui l'union de deux individus. En outre, il était le seul cadre susceptible d'accueillir la famille légitime qui avait la faveur du législateur.
Cependant, il n'existe plus désormais de différence de statut entre enfants légitimes et enfants naturels et, en tout état de cause, le lien entre mariage et procréation n'est plus aussi étroit aujourd'hui. L'argument selon lequel deux personnes de même sexe ne peuvent procréer ensemble n'est dès lors nullement probant pour leur interdire le mariage. La jurisprudence de la Cour européenne des droits de l'homme reflète avec une particulière acuité cette évolution : alors qu'en 1986 elle reconnaissait protéger le mariage en tant que fondement de la famille ${ }^{25}$, en 2002 elle affirmait que le droit de fonder une famille «n' [était] pas une condition » du droit de se marier et que «l'incapacité pour un couple de concevoir ou d'élever un enfant ne saurait en soi passer pour le priver du droit [de se marier] ${ }^{26}$. Ainsi, le mariage a surtout des effets spécifiques dans les relations entre époux. Cela apparaît avec une particulière évidence si l'on songe aux régimes matrimoniaux ou aux droits successoraux: on ne peut douter que le mariage ait pour principal corollaire l'établissement de liens pécuniaires entre les époux. Le législateur a d'ailleurs mis l'accent sur cette dimension patrimoniale lors de la récente réforme des droits du conjoint survivant ${ }^{27}$. Quant aux droits et devoirs respectifs des époux, édictés par les articles 212 et suivants du Code civil, ils pourraient fort bien trouver à s'appliquer dans les relations entre deux personnes de même sexe ${ }^{28}$. Il n'est donc pas si aberrant de prétendre qu'un couple d'homosexuels puisse se marier ${ }^{29}$.

Si le droit positif exclut le mariage pour les couples de même sexe, la lecture des dispositions du Code civil et l'évolution de la philosophie de l'institution montrent que le lien entre mariage et hétérosexualité est beaucoup moins évident que l'on pouvait le penser de prime abord. Une approche intuitive laisserait à penser que la filiation est davantage réfractaire à l'homosexualité que le mariage. Ce postulat peut-il également être contredit?

\section{B. La filiation}

Les tribunaux ont régulièrement à établir si l'homosexualité d'un parent est un obstacle à l'attribution de l'autorité parentale. La question se pose également lors de la fixation de la résidence habituelle de l'enfant et du

21. Il est d'ailleurs unanimement enseigné qu'un mariage conclu entre deux hommes ou deux femmes serait nul de nullité absolue, la théorie de l'inexistence, parfois invoquée, semblant quelque peu tombée en désuétude (G. Cornu, Droit civil. La famille, $7^{\mathrm{e}}$ éd., Paris, Montchrestien, $2001, \mathrm{n}^{\circ}$ 194; P. Courbe, Droit de la famille, $\mathrm{n}^{\circ} 355$ ). Tel devrait être le sort du mariage récemment célébré entre deux hommes par N. Mamère, député et maire de Bègles, événement dont la presse s'est abondamment fait l'écho.

22. Également en ce sens, I. Corpart, «L'homosexualité à l'épreuve du droit de la famille», RRJ, 2003, p. 703.

23. Il est en effet peu probable que les juges puissent prendre l'initiative d'ouvrir le mariage aux homosexuels. Certes, ils ont le pouvoir d'interpréter la loi et cette interprétation peut évoluer avec l'esprit du temps. Toutefois, il paraît difficile de passer outre le libellé parfaitement clair de l'article 75 . De surcroît, la question du mariage des homosexuels est une véritable question de société et il serait regrettable que les juges confisquent ce débat.

24. Discours du 16 Ventôse an XI (10 mars 1803) devant le corps législatif.

25. Arrêt Rees c. Royaume-Uni, 25 septembre 1986, série A, nº 106, $\$ 49$.

26. Arrêt Christine Goodwin c. Royaume-Uni, $\$ 98$.

27. Loi ${ }^{\circ}$ 2001-1135 du 3 décembre 2001.

28. On pourrait certes considérer comme problématiques les dispositions faisant référence à l'éducation des enfants (art. 213 et 220 C. civ.). Il convient toutefois de souligner que le libellé de ces articles n'a jamais justifié d'interdire le mariage à des couples hétérosexuels qui ne pouvaient ou ne souhaitaient pas avoir d'enfants. Ces dispositions ne trouvent tout simplement pas à s'appliquer dans ces circonstances. Il en serait de même dans l'hypothèse d'une union entre homosexuels.

29. On pourrait certes songer à invoquer la tradition en la matière. Toutefois, la référence à la tradition (comme d'ailleurs à la nature) n'est jamais probante dans un discours juridique: soutenir le contraire aurait interdit toute réforme des institutions familiales. 
droit de visite et d'hébergement des parents. Il semble éminemment contestable de rompre les liens entre un enfant et son parent au motif que celui-ci est homosexuel. Le choix de vie d'un parent ne rejaillit nullement sur ses qualités éducatives, ni sur les liens affectifs qui l'unissent à ses enfants. La jurisprudence récente est d'ailleurs majoritairement en ce sens ${ }^{30}$. En effet, seuls des motifs graves peuvent justifier le retrait de l'autorité parentale ou le refus d'un droit de visite et, comme l'a expressément mentionné une Cour d'appel, «l'homosexualité n'est en aucune façon susceptible de constituer l'un de ces motifs ${ }^{31}$. Certaines décisions n'hésitent pas à fixer la résidence habituelle de l'enfant chez le père homosexuel, signe s'il en est de l'évolution des mentalités ${ }^{32}$, en se fondant notamment sur le fait que l'enfant lui-même en exprime le désir ${ }^{33}$ ou encore qu'il apparaît parfaitement heureux et épanoui auprès de son père ${ }^{34}$. La jurisprudence française se révèle ici en accord avec la position de la Cour européenne des droits de l'homme: celle-ci considère comme discriminatoire tout refus d'attribuer l'autorité parentale qui serait motivé exclusivement par l'orientation sexuelle du parent ${ }^{35}$.

Une personne homosexuelle est en droit de conserver des relations avec ses enfants. Homosexualité et filiation ne sont donc pas totalement antinomiques. Il est autrement délicat d'établir s'il est loisible aux homosexuels de créer artificiellement un lien de filiation par le recours à l'adoption. On mentionnera ici une hypothèse particulière: celle de l'adoption au sein d'un couple homosexuel ${ }^{36}$. Deux individus de même sexe peuvent en effet être tentés d'établir un lien juridique entre eux en recourant au mécanisme de l'adoption simple ${ }^{37}$. Il est théoriquement concevable, dès lors qu'il existe entre ces personnes une différence d'âge de quinze ans au moins ${ }^{38}$, de requérir l'adoption du plus jeune par le plus âgé. Certaines juridictions ont accepté de consacrer cette pseudo-parenté. La Cour d'appel de Paris, dans un arrêt en date du 2 juillet 1982, s'est contentée de relever que les conditions légales tenant à la différence d'âge et aux consentements étaient remplies et que l'adoption était conforme à l'intérêt de l'adopté $^{39}$, et la Cour de cassation elle-même a semblé admettre, dans un arrêt rendu le 8 juin $1999^{40}$, la validité de ces adoptions, en estimant que le fait que l'adopté ait eu des relations homosexuelles avec l'adoptant ne pouvait constituer une fraude ou un $\mathrm{dol}^{41}$. Il est toutefois certain que ces décisions ont fait preuve d'une méconnaissance du rôle de l'adoption: celle-ci a pour finalité l'établissement d'un lien de filiation. Certes, il s'agit d'un lien électif, mais ce lien est conçu à l'imitation de la filiation biologique. On peut noter en ce sens que les textes relatifs à l'adoption simple exigent une différence d'âge entre adoptant et adopté qui permet de donner l'apparence d'une filiation par le sang ${ }^{42}$ et qu'il est expressément précisé que l'adoption simple crée un véritable lien de parentét ${ }^{43}$. En outre, les effets de l'adoption sont ceux d'une filiation, qu'il s'agisse de l'autorité parentale si l'adopté est mineur ${ }^{44}$ ou des droits successoraux ${ }^{45}$. Admettre l'adoption au sein d'un couple reviendrait ainsi à consacrer un véritable détournement de l'institution de l'adoption, mais aussi à cautionner juridiquement une forme d'inceste symbolique.

30. I. Corpart, «L'homosexualité...», p. 718. L'autorité parentale a même été octroyée à un homme engagé dans une relation homosexuelle qui avait consenti à l'insémination artificielle d'une femme elle-même engagée dans une relation de ce type (Civ. ${ }^{\text {re }}$, 9 mars 1994, PA, 28 septembre 1994, p. 21, note J. Massip et 28 décembre 1994, p. 24, note X. Daverat, D., 1995, p. 197, note E. Monteiro).

31. CA Pau, 19 juin 2000 (Juris-Data $\mathrm{n}^{\circ}$ 2000-122426). En revanche, ces droits parentaux peuvent être notablement restreints, non en raison de l'homosexualité du parent, mais de son comportement ou de ses conditions de vie lorsque ceux-ci peuvent s'avérer dangereux pour l'enfant ou risquent de le perturber (A. Gouron-Mazel, «Juge de la famille... », p. 6 sq.). On peut cependant penser que les restrictions en cause seraient tout aussi justifiées si ce parent était hétérosexuel, les comportements déviants incriminés n'étant pas liés à l'homosexualité elle-même.

32. On sait que la résidence habituelle de l'enfant est plus volontiers fixée chez la mère, indépendamment de tout grief susceptible d'être imputé aux parents.

33. CA Douai, 21 octobre 1993, Gaz. Pal., 1994, 1, somm. p. 74.

34. CA Pau, 25 avril 1991, D., 1993, somm. p. 122, obs. M. Wacongne.

35. Arrêt Salgueiro Da Silva Moura c. Portugal, 21 décembre 1999, req. nº 93290 / 96, Dr fam., nº 45, 2000, note A. Gouttenoire-Cornut, Rev. trim. Dr civ., 200o, p. 313, obs. J. Hauser.

36. Cette pratique n'est pas spécifique aux couples homosexuels. L'un des premiers arrêts cités comme ayant consacré une adoption au sein d'un couple concernait d'ailleurs deux concubins hétérosexuels (CA Riom, 9 juillet 1981, JCP Ed. G., 1982, II 19799, note G. Almairac). Toutefois, avant la consécration du PACS, elle était la seule possibilité d'établir entre eux un lien juridique. Ils y ont donc plus volontiers eu recours que les couples hétérosexuels.

37. On pourrait penser de prime abord que l'objectif de ces adoptions est d'éluder le paiement de droits de mutation. Or, il s'avère qu'elles ne présentent aucun intérêt fiscal, le Code général des impôts exigeant, pour qu'il soit tenu compte du lien de parenté dans cette hypothèse, que l'adopté ait «soit dans [sa] minorité et pendant six ans au moins, soit dans [sa] minorité et [sa] majorité et pendant dix ans au moins, reçu de l'adoptant des secours et des soins non interrompus» (art. 786), condition qui peut difficilement être remplie dans ces circonstances. Le seul intérêt patrimonial pourrait être de contourner partiellement les règles de la réserve héréditaire si l'adoptant a des héritiers susceptibles d'y prétendre. À cela s'ajoute l'intérêt plus symbolique, parfois invoqué, de permettre aux deux membres du couple de porter le même nom (sic).

38. Art. 344 C. civ. Une dérogation à cette condition d'âge peut être accordée par le tribunal «s'il y a de justes motifs ", condition qui pourra difficilement être remplie dans ces hypothèses.

39. CA Paris, 2 juillet 1982, annexe à l'article de P. Raynaud, «Un abus de l'adoption simple. Les couples adoptifs », D., 1983 , p. 39.

40. Civ. $1^{\text {re }}, 8$ juin 1999, Dr fam., $\mathrm{n}^{\circ}$ 124, 1999, note P. Murat, RJPF, 2000, 1/36, Rev. trim. Dr civ., 1999, p. 610, obs. J. Hauser.

41. On remarquera que cet arrêt avait semblé ressusciter la notion de bonnes mœurs telle qu'elle résultait de la jurisprudence relative aux libéralités entre concubins : pour justifier le rejet de la tierce opposition, il approuvait la Cour d'appel d'avoir jugé « qu'il n'était pas établi que l'adoption avait été sollicitée pour permettre la création de relations homosexuelles, ni même pour les favoriser ou les consacrer». Cette motivation apparait toutefois éminemment contestable.

42. Art. 344 C. civ.

43. Art. 366 C. civ.

44. Art. 365 C. civ.

45. Art. 368 C. civ. 
En dehors de ces hypothèses d'adoption frauduleuse, la véritable question est celle du désir des homosexuels d'adopter des enfants. L'adoption n'est pas ouverte aux concubins : elle est réservée aux couples mariés. En l'état du droit positif, un couple d'homosexuels ne peut donc adopter conjointement un enfant. En revanche, l'adoption peut être demandée à titre individuel par toute personne âgée de plus de vingt-huit ans ${ }^{46}$. Il est donc juridiquement envisageable qu'une personne homosexuelle sollicite une adoption. Cependant, le Conseil d'État se révèle radicalement opposé à la délivrance d'un agrément à l'adoption dans ces circonstances ${ }^{47}$. On peut mentionner comme particulièrement significatif l'arrêt du Conseil d'État du 9 octobre 1996, rendu dans la désormais célèbre affaire Fretté $^{48}$, qui a considéré qu'un célibataire homosexuel, « eu égard à ses conditions de vie, et malgré des qualités humaines et éducatives certaines, ne présentait pas de garanties suffisantes sur les plans familial, éducatif et psychologique pour accueillir un enfant adopté». Certes, le Conseil d'État n'énonce pas clairement que l'homosexualité est un obstacle rédhibitoire à l'adoption et se retranche derrière les conditions de vie du demandeur. Toutefois, les conclusions du commissaire du gouvernement établissent sans ambiguïté que c'est l'homosexualité du demandeur qui a justifié la décision ${ }^{49}$ et l'étude de la jurisprudence montre que les juges estiment que les conditions d'accueil d'un enfant ne peuvent jamais être considérées comme satisfaisantes si le demandeur est homosexuel, la situation étant d'ailleurs la même que l'intéressé soit un homme ou une femme ${ }^{50}$. La Cour européenne des droits de l'homme, qui a été saisie d'un recours à la suite de l'arrêt de $1996^{51}$, ne s'y est d'ailleurs pas trompée et a énoncé que cette décision reposait de manière déterminante sur l'homosexualité déclarée du requérant $^{52}$. Toutefois, la Cour n'a pas sanctionné la France au motif que sur cette question une grande marge d'appréciation devait être laissée aux États ${ }^{53}$ et qu'il n'y avait pas discrimination dans la mesure où la distinction en cause poursuivait un but légitime ("protéger la santé et les droits des enfants pouvant être concernés par une procédure d'adoption ${ }^{54}$ ) et où elle n'était pas disproportionnée par rapport à cet objectif ${ }^{55}$. Il apparaît ainsi que seul l'intérêt de l'enfant peut justifier l'exclusion des homosexuels de l'adoption. La jurisprudence administrative poserait alors une présomption irréfragable selon laquelle un homosexuel est incapable d'élever un enfant, ce qui est en totale contradiction avec les dossiers rejetés, qui insistent sur les qualités humaines et éducatives des demandeurs ${ }^{56}$, ainsi qu'avec les décisions qui fixent la résidence habituelle des enfants chez leur parent homosexuel. Si les qualités de ces personnes ne sont pas en cause, en quoi l'adoption par un homosexuel pourraitelle être contraire à l'intérêt de l'enfant à adopter? On remarque que les juges se retranchent souvent derrière l'absence de référent paternel ou maternel, alors que l'enfant aurait besoin, pour sa construction psychologique, d'une image de l'altérité sexuelle ${ }^{57}$. Cet argument se réclame de théories psychanalytiques. Toutefois, on peut douter de l'opportunité de laisser les individus à la merci d'un "freudisme administratif » ${ }^{58}$ parfois mal maîtrisé. De surcroît, cette question de l'altérité sexuelle trouve difficilement sa place dans un discours juridique alors que l'adoption par un célibataire est permise et que, qui plus est, les juges sanctionnent les refus d'agrément motivés par le seul célibat des demandeurs ${ }^{59}$. Seraient en revanche plus à même de justifier les refus d'agrément les réactions

46. Art. 343-1 C. civ.

47. Cet agrément est requis de ceux qui désirent adopter un pupille de l'État (art. 63 C. fam.) ou un enfant étranger (art. 100-3 C. fam.). Il est accordé par le Président du Conseil général après avis d'une commission.

48. CE 9 octobre 1996, JCP Ed. G., 1997, II 22766, concl. C. Maugüé, Rep. Not. Def., 1997, p. 726, obs. J. Massip, D., 1997, p. 117, note P. Malaurie, PA, 11 juillet 1997, p. 32, note J. Massip, Rev. trim. Dr civ., 1997, p. 408, obs. J. Hauser.

49. Il est en effet expressément précisé que «le seul élément qui a conduit l'Administration à refuser l'agrément est le fait que [le demandeur] est homosexuel ». Le commissaire du gouvernement reconnaît ensuite qu' «admettre la légalité du refus d'agrément dans le cas présent revient à condamner implicitement mais nécessairement à l'échec toute demande d'agrément en vue de l'adoption émanant d'un homosexuel».

50. CAA Lyon, 7 juillet 1999, AJDA, 1999, p. 1033, concl. A. Bézard; CAA Nancy, 21 décembre 2000, AJDA, 2001, p. 295, concl. P. Rousselle, D., 2001, p. 1575, note R. Piastra ; CE 5 juin 2002, AJDA, 2002, p. 615, concl. P. Fombeur, D., 2002, IR, p. 2025, RJPF, 2002, 10/30, Rev. trim. Dr civ., 2002, p. 496, obs. J. Hauser et p. 611, obs. R. Libchaber.

51. Arrêt Fretté c. France, 26 février 2002, req. $\mathrm{n}^{\circ}$ 36515/97, PA, 10 juillet 2002, p. 10, note F. Vasseur-Lambry et M. Carius, JCP Ed. G., 2002, II 10 074, note A. Gouttenoire-Cornut, Rev. trim. Dr civ., 2002, p. 280, obs. J. Hauser et p. 389, obs. J.-P. Marguenaud; B. Bourdelois, «Vie privée et familiale et nondiscrimination : l'adoption par un homosexuel (arrêt Fretté du 26 février 2002) ", in La France et la Cour européenne des Droits de l'Homme. La jurisprudence en 2002, P. Tavernier (dir.), Cahiers du CREDHO, nº 9, 2003, p. 165.

52. $\$ 32$.

53. $\$ 41$.

54. $\$ 38$.

55. $\$ 42$.

56. Dans ses conclusions sur l'arrêt du Conseil d'État du 9 octobre 1996, le commissaire du gouvernement notait que les rapports extrêmement élogieux concernant le demandeur le décrivaient comme « un homme cultivé, travailleur, sensible, réfléchi, attentif aux autres, constant dans ses amitiés, scrupuleux, altruiste». Il n'était par ailleurs formulé «aucun doute quant à ses qualités humaines indiscutables et une sensibilité exacerbée confirmée par sa qualité d'enseignant [...]. Son sens de la pédagogie et son tact sont à cet égard bien connus ». De surcroît, il était précisé : « [le demandeur] possède des qualités humaines et éducatives certaines. Un enfant serait probablement heureux avec lui.» Il n'est pas certain que toutes les personnes agréées par l'administration puissent faire état d'autant de qualités.

57. CE 5 juin 2002.

58. L'expression est de P. Hubert, concl. sur CE 4 novembre 1991, AJDA, 1992, p. 73.

59. CE 4 novembre 1991, AJDA, 1992, p. 65, concl. P. Hubert; CE 24 avril 1992, Rev. adm., 1992, p. 328, obs. H. Ruiz-Fabri (le dossier en l'espèce faisait pourtant état de «tendances homosexuelles refoulées»). 
de rejet que pourrait subir l'enfant dans son milieu social en raison de l'homosexualité de son parent adoptif. Il est soutenu que l'on ne peut imposer cette difficulté supplémentaire à un enfant qui a déjà subi le traumatisme de l'abandon et qui doit faire face aux difficultés de la transplantation dans une nouvelle famille ${ }^{60}$. Cet argument semble davantage probant que le précédent. Toutefois, l'admettre sans mesure reviendrait à interdire l'adoption à tout membre d'une minorité car il y aurait un risque réel de discrimination pour l'enfant. Or, les tribunaux n'ont jamais prétendu se résoudre à une telle extrémité. Il faudrait alors en déduire que cet argument ne vaut que pour les homosexuels, ce qui serait un regrettable constat d'échec pour notre société, puisque cela signifierait que les homosexuels sont tellement stigmatisés que leur entourage lui-même subit les conséquences du rejet dont ils sont l'objet. On peut hésiter à adhérer à cette vision pessimiste de la condition des homosexuels en France. En outre, il convient de souligner que l'effet pervers de la jurisprudence actuelle est d'inciter à l'hypocrisie et au mensonge puisque la personne qui dissimulera son homosexualité pourra en toute quiétude se voir délivrer un agrément. Il serait plus satisfaisant de suivre la voie ouverte par certains juges du fond qui avaient admis qu'un agrément puisse être délivré à un homosexuel dès lors qu'il présentait des qualités humaines et éducatives certaines et qu'il était bien inséré dans son milieu social ${ }^{61}$.

Si les institutions familiales peuvent sembler réfractaires à l'homosexualité, il s'avère que cette hostilité est plus apparente que réelle. La perspective ne serait-elle pas dès lors celle d'une prise en compte explicite des homosexuels par ces institutions?

\section{Une évolution inéluctable ? [Étude de droit prospectif]}

L'évolution des législations étrangères et la jurisprudence de la Cour européenne des droits de l'homme sont de nature à inciter le législateur français à ouvrir le mariage, sinon l'adoption ${ }^{62}$, aux couples homosexuels (A). Il apparaît dès lors plus satisfaisant de prendre l'initiative d'une réforme nationale mesurée, plutôt que d'attendre d'y être contraint (B).

\section{A. L'existence de pressions extérieures}

D'autres législations ont ouvert le mariage aux homosexuels : tel est le cas de la Belgique ${ }^{63}$ et des Pays-Bas ${ }^{64}$. La Suède et l'Espagne y songent également. La proximité de ces pays induit nécessairement que les juges français auront à connaître des effets de telles unions. Celles-ci seront-elles considérées comme valables? On pourrait envisager que l'ordre public français s'oppose à l'application d'une loi autorisant le mariage entre homosexuels ${ }^{65}$, mais cette affirmation gagnerait à être nuancée. Certes, l'exception d'ordre public sera indéniablement opposée à toute tentative de célébration en France d'un mariage entre deux personnes de même sexe ${ }^{66}$, quand bien même leurs lois nationales respectives l'autoriseraient ${ }^{67}$. Toutefois, la question se poserait sans doute différemment s'il s'agissait simplement de reconnaître les effets en France d'un mariage valablement célébré à l'étranger. Dans de telles circonstances, la théorie de l'effet atténué de l'ordre public trouverait certainement à s'appliquer ${ }^{68}$. Depuis

60. Les conclusions précitées du commissaire du gouvernement dans l'affaire Fretté sont en ce sens: «L'enfant adopté a d'autant plus besoin d'avoir un environnement stable et épanouissant qu'il a été privé de sa famille d'origine et a déjà un passé douloureux: il importe donc tout particulièrement qu'il ne trouve pas une difficulté supplémentaire dans son milieu d'adoption. »

61. TA Paris, 25 janvier 1995, D., 1995 p. 647, note F. Boulanger, PA, 30 juin 1995, p. 20, note J.-Y. Plouvin; TA Besançon, 24 février 2000, Rev. Dr sanit. et soc., 2000, p. 434, obs. F. Monéger.

62. Les Pays-Bas ont ouvert l'adoption aux couples homosexuels. Toutefois, cette question se pose avec moins d'acuité que celle du mariage, du moins pour le moment. La prise de position de cet État semble en effet isolée, pour ne pas dire marginale, et la possibilité d'adoption ouverte par la loi a un champ d'application restreint puisqu'elle ne peut concerner que des enfants néerlandais. La Belgique qui, à sa suite, a admis le mariage de deux personnes de même sexe, a néanmoins exclu les questions de filiation. De surcroît, comme il a été mentionné précédemment, la Cour européenne des droits de l'homme ne semble nullement disposée à reconnaître le droit pour les homosexuels de recourir à l'adoption, ne serait-ce qu'à titre individuel. Quant à la question de l'adoption conjointe, elle ne lui a pas encore été soumise. Ainsi, bien que la Cour ait observé que, sur cette question, le droit paraissait «traverser une phase de transition » (arrêt Fretté c. France, $\$ 44$ ), il est probable que cette phase se révèle longue et semée d'embûches.

63. Loi du 13 février 2003, entrée en vigueur le $1^{\text {er }}$ juin 2003.

64. Loi du 21 décembre 2000, entrée en vigueur le $1^{\mathrm{er}}$ avril 2001.

65. En ce sens, H. Fulchiron, «Le mariage homosexuel et le droit français (à propos des lois hollandaises du 21 décembre 2001) », D., 2001, p. 1628.

66. L'ordre public sera en tout cas invoqué par le Ministère public, car l'actualité récente révèle que certains officiers publics acceptent de prêter leur concours à de telles célébrations.

67. On peut hésiter à considérer que le « mariage homosexuel » puisse poser, en amont de la désignation de la loi applicable, un problème de qualification. Il a certes pu être soutenu que «dans le cas du mariage entre personnes de même sexe, l'essence même du mariage est en cause. L'élargissement de la catégorie mariage n'est plus possible car on touche à la nature du mariage qui se définit comme l'union de l'homme et de la femme» (H. Fulchiron, «Le mariage homosexuel... », p. 1629). Il est indéniable que le «mariage homosexuel» est inconnu du droit français, mais, pour qualifier une institution inconnue de la lex fori, il convient de se référer à la loi de cette institution (CA Alger, 24 décembre 1889, Veuve Bartholo, JDI, 1891 p. 1171, B. Ancel et Y. Lequette, Les Grands Arrêts de la jurisprudence de droit international privé, $4^{e}$ éd., Paris, Dalloz, 2001, nº 9, p. 73). Or, il ne fait nul doute que le mariage entre homosexuels est considéré comme un véritable mariage par les États qui le reconnaissent. Dès lors, puisqu'il s'agit bien d'un mariage et que la différence de sexe peut être qualifiée de condition de fond, la règle de conflit de lois française renvoie à la loi nationale des époux (art. 3 al. 3 C. civ.). Il sera aisé de trancher si les lois nationales des époux interdisent ou autorisent toutes deux le mariage entre personnes de même sexe. Plus problématique apparaît l'hypothèse où les époux sont ressortissants d'États différents, dont l'un admet le «mariage homosexuel» et l'autre non. La différence de sexe exigée par la loi prohibitive peut être considérée comme une condition bilatérale, concernant les deux époux. Il conviendra alors d'appliquer cumulativement les deux lois : par conséquent le mariage ne sera valable que si les deux lois nationales admettent le mariage entre homosexuels.

68. Contra: H. Fulchiron, «Le mariage homosexuel...», p. 1630. 
l'arrêt Rivière ${ }^{69}$, il est établi que « la réaction à l'encontre d'une disposition contraire à l'ordre public n'est pas la même suivant qu'elle met obstacle à l'acquisition d'un droit en France ou suivant qu'il s'agit de laisser se produire en France les effets d'un droit acquis sans fraude à l'étranger et en conformité de la loi ayant compétence en vertu du droit international privé français ». Cette distinction de l'effet plein et de l'effet atténué de l'ordre public ${ }^{70}$ a permis par exemple de reconnaître des effets à des unions polygamiques, tels que des droits successoraux ou des obligations alimentaires ${ }^{71}$, alors qu'un mariage polygamique ne pourrait être valablement célébré sur le territoire français. La même distinction présiderait à la reconnaissance en France des effets d'unions homosexuelles valablement célébrées à l'étranger: il ne semble pas que le mariage de personnes de même sexe heurte davantage les principes fondamentaux du droit français que la polygamie. Comment justifier la différence de traitement qui serait alors faite entre les ressortissants d'États acceptant de telles unions et les Français ? D’aucuns seront choqués par cette discrimination qui autorise un couple d'homosexuels néerlandais vivant en France à se prévaloir de leur condition d'époux alors que leurs voisins français se voient interdire l'accès au mariage. On pourrait certes arguer du fait que l'effet atténué de l'ordre public n'a jamais incité le législateur à autoriser aux Français l'union polygamique. Toutefois, la question ne se pose pas exactement dans les mêmes termes. Tout d'abord, les pays qui admettent le mariage des homosexuels gravitent dans le même espace culturel que la France et l'ouverture du mariage aux couples de même sexe semble aller dans le sens de l'évolution actuelle des législations européennes ${ }^{72}$. Ensuite, le mariage polygamique nie l'égalité entre hommes et femmes, ce qui heurte particulièrement les principes républicains, alors que les unions homosexuelles ne sont nullement en contradiction avec ces principes. Enfin, il existe un risque de voir se multiplier les situations «boiteuses», celles de Français ${ }^{73}$ valablement mariés aux Pays-Bas mais simples concubins en France. En effet, les règles de conflit de lois adoptées aux Pays-Bas précisent que le mariage qui y est célébré est valable dès lors que l'un des époux a la nationalité néerlandaise ou réside aux Pays-Bas. Il est de surcroît précisé que, dans cette hypothèse, les conditions de validité du mariage sont régies par la loi néerlandaise ${ }^{74}$. Deux Français de même sexe pourraient ainsi fort bien se marier aux Pays-Bas dès lors que l'un d'eux y a établi sa résidence. Or, s'ils établissent par la suite leur résidence commune en France, ils y seront considérés comme célibataires. Cette situation semble fort peu satisfaisante ${ }^{75}$.

Et surtout, ne peut-on pas voir dans la jurisprudence de la Cour européenne des droits de l'homme les prémices de la consécration d'un droit au mariage pour les homosexuels ? On a naturellement à l'esprit les arrêts rendus le 11 juillet $2002^{76}$. La Cour y a considéré que le RoyaumeUni ne pouvait interdire à un transsexuel d'épouser une personne de son sexe d'origine. Elle admet donc bien que deux personnes de même sexe chromosomique puissent se marier : on est ici à la frontière du mariage homosexuel. Certes, le transsexuel, pour se marier, doit avoir subi des opérations chirurgicales de nature à lui conférer certaines caractéristiques du sexe opposé. Toutefois, les arguments avancés par la Cour pourraient fort bien justifier l'instauration d'une union homosexuelle. La Cour a en effet réfuté comme artificielle l'affirmation selon laquelle les transsexuels auraient le droit de se marier puisqu'il leur serait possible d'épouser une personne du sexe opposé à leur ancien sexe ${ }^{77}$. Tout aussi fallacieux serait alors l'argument selon lequel les homosexuels peuvent contracter mariage avec des individus du sexe opposé. La Cour a ajouté que la requérante « entret[enait] une relation avec un homme et souhait[ait] épouser uniquement un homme. Or elle n'en a[vait] pas la possibilité». Pour la Cour, l'intéressée « $\mathrm{p}$ [ouvait] donc se plaindre d'une atteinte à la substance même du droit de se marier ${ }^{78}$. Le raisonnement vaut également pour les homosexuels. De surcroît, la Cour s'est référée explicitement à l'article 9 de la Charte des droits fondamentaux de l'Union européenne ${ }^{79}$ et a précisé que, si cette disposition excluait, à la différence de l'article 12 de la Convention européenne des droits de l'homme $^{80}$, la référence à l'homme et à la femme, «cela

69. Civ. 17 avril 1953, Rev. crit. Dr int. pr., 1953, p. 412, note Batiffol, JDI, 1953, p. 860, note Plaisant, JCP, 1953, II 7863, note Buchet, B. Ancel et Y. Lequette, Les Grands Arrêts..., n 26.

70. Sur cette notion, voir P. Mayer et V. Heuzé, Droit international privé, $7^{\mathrm{e}}$ éd., Paris, Montchrestien, 2001, $\mathrm{n}^{\circ} 206 \mathrm{sq}$.

71. Civ. 28 janvier 1958, Rev. crit. Dr int. pr., 1958, p. 110, note Jambu-Merlin, D., 1958, p. 265, note Lenoan, JCP, 1958, II 10 488, note Louis-Lucas, JDI, 1958, p. 776, note Ponsard; Civ. 19 février 1963, Rev. crit. Dr int. pr., 1963, p. 559, note G. H., JDI, 1963, p. 986, note Ponsard; Civ. $1^{\text {re }}, 3$ janvier 1980, Rev. crit. Dr int. pr., 1980, p. 331, note Batiffol, JDI, 1980, p. 327, note Simon-Depitre, D., 1980, p. 549, note Poisson-Drocourt; B. Ancel et Y. Lequette, Les Grands Arrêts..., $\mathrm{n}^{\circ} 30-31$, p. 276 et n ${ }^{\circ} 61$, p. 572.

72. On a au contraire pu souligner «l'absence de communauté de civilisation entre les systèmes juridiques qui connaissent la polygamie et ceux qui l'ignorent» (B. Ancel et Y. Lequette, Les Grands Arrêts..., p. 382).

73. Ou de ressortissants d'autres États prohibant le mariage entre personnes de même sexe.

74. H. Fulchiron, «La reconnaissance de la famille homosexuelle aux Pays-Bas», JCP Ed. G., 2001, actualité, p. 1034.

75. Le problème semble se poser de façon moins cruciale s'agissant de la reconnaissance de ces mariages par la Belgique. En effet, la règle de conflit de lois belge, à l'instar de la règle française, donne compétence aux lois nationales des époux pour régir les conditions de fond du mariage ( $\mathrm{M}$. Schmitt, "L’incidence en France des lois belge et néerlandaise introduisant le mariage homosexuel», JCP Ed. G., 2004, p. 1006). Les juges belges feront donc vraisemblablement une application cumulative des deux lois nationales en cause.

76. Arrêts Christine Goodwin c. Royaume-Uni, req. nº 28957/95 et I. c. Royaume-Uni, req. nº 25680/94, rendus le 11 juillet 2002, D., 2002, IR, p. 2305 , D., 2003, p. 2032, note A.-S. Chavent-Leclère, JCP Ed. G., 2003, I 101, obs. J. Rubellin-Devichi, AJDA, 2002, p. 1277, obs. J.-F. Flauss.

77. Arrêt Christine Goodwin c. Royaume-Uni, \$101.

78. $\$ 101$.

79. «Le droit de se marier et le droit de fonder une famille sont garantis selon les lois nationales qui en régissent l'exercice».

80. «À partir de l'âge nubile, l'homme et la femme ont le droit de se marier et de fonder une famille selon les lois nationales régissant l'exercice de ce droit». 
ne $\mathrm{p}$ [ouvait] être que délibéré ${ }^{81}$ : la porte est grande ouverte au mariage des homosexuels. On ajoutera que la Cour revendique une interprétation évolutive de la Convention européenne des droits de l'homme, laquelle « est un instrument vivant à interpréter à la lumière des conditions actuelles ${ }^{82}$. L'évolution des législations européennes, et plus largement de l'opinion publique, seront alors déterminantes. Si la Cour a pu attacher de l'importance à l'existence d'une « tendance internationale continue, non seulement vers une acceptation sociale accrue des transsexuels, mais aussi vers la reconnaissance juridique de la nouvelle identité sexuelle des transsexuels opérés ${ }^{83}$, le statut des couples homosexuels, mieux acceptés par le droit et accédant d'ores et déjà à une certaine reconnaissance juridique, devra donc être prochainement reconsidéré.

Il apparaît alors souhaitable de prendre l'initiative d'un véritable débat national sur l'opportunité d'une modification du droit positif, plutôt que d'attendre passivement une condamnation de la Cour européenne des droits de l'homme. On peut souligner à cet égard que, comme la Cour l'a elle-même admis ${ }^{84}$, sur des questions aussi sensibles les autorités nationales sont les mieux à même d'évaluer la réalité et la légitimité des revendications existantes et de déterminer, à l'issue de véritables négociations, les moyens les plus appropriés pour y répondre.

\section{B. La nécessité de contrôler l'évolution}

Le Pacte civil de solidarité pourrait-il constituer une alternative au mariage des homosexuels ? Il sera sans doute soutenu qu'axer le débat sur le PACS permet de cantonner les homosexuels en marge du droit de la famille: on se souvient qu'il a été affirmé lors de l'adoption de la loi de 1999 que le PACS n'interférerait nullement avec le droit de la famille et, en effet, il n'a aucune incidence sur la filiation ou l'état des personnes ${ }^{85}$. Toutefois, le Conseil constitutionnel en a donné une lecture quelque peu «familiale», qui montre qu'il ne s'agit pas seulement d'établir un lien patrimonial entre les partenaires ${ }^{86}$. Aussi la vie commune organisée par le PACS ne se limite-t-elle pas à une communauté d'intérêts, ni même à une cohabitation. Elle suppose au contraire, selon les termes du Conseil constitutionnel, une véritable «vie de couple». C'est d'ailleurs l'existence de cette communauté de vie qui justifie les empêchements à la conclusion d'un $\mathrm{PACS}^{87}$, empêchements qui ne sont pas sans rappeler ceux qui existent en matière de mariage ${ }^{88}$ et que l'on justifie traditionnellement par la prohibition de l'inceste et de la bigamie $^{89}$. En outre, le droit du travail associe clairement PACS et famille puisque les partenaires peuvent bénéficier des congés pour événements familiaux et des dispositions relatives au rapprochement des familles ${ }^{90}$. Le législateur a donc bien consacré une nouvelle forme de conjugalité et non un simple contrat de nature patrimoniale ${ }^{91}$. Cependant, il conviendrait de remédier aux insuffisances du PACS. L'organisation pécuniaire de la vie de couple qu'il instaure apparaît en effet quelque peu déficiente. L'indivision prévue pour la gestion des biens des partenaires ${ }^{92}$ est plus appropriée pour régir une situation temporaire, suspendue à la possibilité d'un partage ${ }^{93}$, que pour l'organisation d'une vie commune susceptible de s'inscrire dans la durée. Il faut ajouter que tout acte d'administration ou de disposition requiert le consentement de chaque indivisaire $^{94}$, condition qui se révèle en pratique extrêmement contraignante $^{95}$. Il peut de surcroît paraître inique qu’il ne soit « pas possible de prévoir contractuellement et une fois pour toutes la disparition de la règle d'indivision ${ }^{96}$. Quant aux règles fiscales applicables au PACS, elles ne permettent aucunement une véritable transmission de patrimoine au partenaire survivant ${ }^{97}$, transmission que

81. $\$ 100$.

82. Arrêt Fretté c. France, $\$ 34$

83. Arrêt Christine Goodwin c. Royaume-Uni, $\$ 85$.

84. Arrêt Fretté c. France, $\$ 41$ : «Étant en prise directe et permanente avec les forces vitales de leur pays, les autorités nationales sont en principe mieux placées qu'une juridiction internationale pour évaluer les sensibilités et le contexte locaux».

85. On peut souligner néanmoins que les articles relatifs au PACS, ainsi d'ailleurs que ceux qui traitent du concubinage, figurent dans le livre I ${ }^{\text {er }}$ du Code civil consacré aux personnes, ce qui montre que l'aspect patrimonial n'est pas nécessairement le plus significatif.

86. Décision du 9 novembre 1999, PA, 26 juillet 2000, p. 11, note B. Mathieu et M. Verpeaux.

87. Un PACS ne peut en effet être conclu entre ascendants et descendants en ligne directe, ni entre alliés en ligne directe et entre collatéraux jusqu'au troisième degré inclus. Il en est de même si l'un des partenaires est marié ou déjà lié par un PACS (art. 515-2 C. civ.).

88. Art. 147 et 161 à 163 C. civ.

89. G. Cornu, Droit civil..., $\mathrm{n}^{\circ} 178$.

90. F. Monéger, «Pacte civil de solidarité (PACS). Aspects sociaux», JCP Ed. N., 2000, p. 452

91. Une ordonnance du président du Tribunal de grande instance de Lille est allée jusqu'à assortir le PACS d'une véritable obligation de fidélité et a qualifié le manquement à cette obligation d'adultère (TGI Lille, 5 juin 2002, D., 2003, p. 515, note X. Labbée, Rev. trim. Dr civ., 2003, p. 270, obs. J. Hauser). Si ce qualificatif est manifestement erroné et si l'on peut hésiter à voir dans l'obligation de fidélité le corollaire nécessaire de la communauté de vie exigée par les textes, il n'en demeure pas moins que cette décision révèle que les juridictions analysent le PACS comme une structure familiale.

92. Art. 515-5 C. civ.

93. Art. 815 C. civ.

94. Art. 815-3 C. civ

95. Il est certes possible aux partenaires de recourir à la technique du mandat pour passer seuls des actes d'administration ou de disposition, mais cette solution n'est pas nécessairement appropriée.

96. F. Courtray, «Pacte civil de solidarité: une occasion manquée», Rev. Dr sanit et soc., 2000, p. 16, pour qui, « en optant pour un régime d'indivision, le PACS emporte des effets pour le moins communautaristes, à l'inverse des tendances sociales actuelles qui font de la liberté individuelle et du droit de propriété exclusif des valeurs fondamentales».

97. Art. 777 bis et 779 III CGI. 
l'on peut pourtant considérer comme une continuité de la vie commune organisée par ce pacte. Semblerait dès lors devoir être modifiée la fiscalité des donations et legs, qui pourrait être alignée sur celle du mariage. Il conviendrait en outre de supprimer l'exigence d'un PACS conclu depuis plus de deux ans pour que s'applique l'abattement prévu pour les donations entre partenaires ${ }^{98}$. Il serait également envisageable de prévoir une alternative à l'indivision légale édictée par le Code civil et de permettre aux partenaires d'adopter, s'ils le souhaitent, un régime des biens qui pourrait s'inspirer de l'un des régimes matrimoniaux existants. Le libre choix du « régime pacsimonial » rendrait là encore le PACS plus intéressant, dans la mesure où l'indivision prévue par le droit positif ne serait plus le seul régime envisageable ${ }^{99}$. Le PACS serait alors plus attractif, non seulement pour les homosexuels, mais aussi pour les hétérosexuels qui souhaiteraient adopter un statut juridique moins contraignant que le mariage.

Il ne s'agit toutefois que d'adaptations ponctuelles et non d'une réelle réforme. Celle-ci, il ne faut pas se le cacher, résiderait inéluctablement dans la reconnaissance du droit au mariage pour les couples de même sexe. Comme on l'a vu, le Code civil serait aisé à modifier et la philosophie de l'institution n'en souffrirait pas autant que l'on pouvait le penser de prime abord ${ }^{100}$. Mais ce droit au mariage, s'il devait être admis, devrait être encadré par des règles ad hoc, à l'instar de ce qu'a décidé le législateur belge : l'exclusion de la filiation des effets d'un tel mariage s'impose. Il est en effet totalement artificiel d'appliquer à un couple de même sexe la présomption de paternité liée au mariage, présomption qui repose sur l'apparence d'une réalité biologique ${ }^{101}$. Il serait également contestable de permettre à deux époux de même sexe de recourir à la procréation médicalement assistée. Celle-ci a en effet pour finalité de remédier à une stérilité pathologique et il ne semble pas souhaitable que la médecine soit utilisée pour satisfaire des convenances personnelles. Quant à la question de l'adoption par un couple homosexuel, elle divise l'opinion davantage encore que le mariage. Il paraît dès lors opportun, sinon d'exclure, du moins de différer toute réforme législative relative à cette question ${ }^{102}$. On peut se donner le temps de réfléchir à ce que sera la famille de demain. L'urgence est, en revanche, de trouver un traitement satisfaisant aux situations existantes et d'accepter de reconnaître un statut aux homosexuels qui, d'ores et déjà, élèvent des enfants qui leur sont juridiquement étrangers. L'adoption simple peut constituer une réponse pertinente à ces questions. L'exemple en a été donné par un jugement du Tribunal de grande instance de Paris en date du 27 juin 2001 qui a admis l'adoption simple de trois enfants par la partenaire de leur mère ${ }^{103}$. Ici, les enfants se voient reconnaître un lien de filiation avec les deux membres du couple homosexuel qui les élèvent. Le tribunal a justifié sa décision par la considération que l'adoption « [aurait] pour résultat de resserrer davantage les liens d'affection qui existent entre [la demanderesse] et les enfants ». On peut également et surtout arguer du fait que cette décision permet de reconnaitre un statut juridique à la personne qui se comporte comme parent de fait. On rappellera ici que la parentalité est une fonction sociale et culturelle et qu'elle ne se limite pas, loin s'en faut, au lien biologique. Or, la filiation adoptive est une filiation élective, qui reflète le véritable choix d'exercer une fonction parentale. Il paraît donc légitime que le droit prenne acte de la réalité quotidienne de ces enfants ${ }^{104}$. On peut également mentionner l'intérêt fiscal de cette décision s'agissant des droits successoraux des enfants ${ }^{105}$ et le fait que cette solution semble à même de prévenir

98. Le montant de cet abattement pourrait également être aligné sur celui dont bénéficient les époux. En revanche, il ne semble pas nécessaire de conférer des droits successoraux au partenaire survivant, et encore moins d'en faire un héritier réservataire, à l'instar du conjoint survivant. Il semble en effet plus opportun d'accorder une grande place à la volonté individuelle, et de laisser chaque partenaire décider librement d'instaurer, ou non, son compagnon comme légataire. Le PACS serait alors, de ce point de vue, plus attractif que le mariage puisqu'il laisserait un véritable choix aux partenaires.

99. On pourrait également envisager des réformes plus ponctuelles: celle des conditions d'imposition commune, celle-ci devenant effective dès la première année et non à compter du troisième anniversaire de l'enregistrement du PACS (art. 6 CGI), ou encore l'octroi de l'assurance vieillesse au partenaire survivant.

100. On ne peut toutefois se départir de l'impression que la revendication du mariage par les homosexuels est, au XXI siècle, quelque peu archaïque. Elle donne de surcroît la fâcheuse impression que la reconnaissance sociale ne peut passer que par le mariage. Parodiant Simone de Beauvoir, qui avait pu dire que, dans les années soixante-dix, le mariage n'était plus qu'une revendication des prêtres, on pourrait affirmer qu'aujourd'hui seuls les homosexuels aspirent au mariage.

101. Art. 312 C. civ. : «L'enfant conçu pendant le mariage a pour père le mari. » De surcroît, cette présomption, faisant référence au mari, ne pourrait jamais trouver à s'appliquer dans un couple de femmes et serait d'application complexe dans un couple d'hommes (il serait seulement envisageable que l'enfant dont l'un serait le père biologique soit présumé être également le fils de l'autre. Ce serait alors faire bien peu de cas de la mère biologique).

102. Il est en effet préférable de procéder par étapes. On se souvient que le projet de loi relatif au PACS avait suscité des débats enflammés. Or, aujourd'hui, plus personne ne songe à en remettre en cause l'existence et il est véritablement entré dans les mœurs. Quant au débat qui s’instaure aujourd'hui sur l'ouverture du mariage aux couples homosexuels, il aurait été inconcevable en 1999. On peut alors supposer que, lorsque le mariage homosexuel ne surprendra plus, le débat pourra s'engager plus sereinement sur les questions d'adoption. S'il était alors établi qu'il existait une réelle revendication par les homosexuels d'une possibilité d'adoption conjointe (mais cette revendication ne serait le fait que d'une infime minorité: B. Bourdelois, «Vie privée... », p. 165), il conviendrait d'envisager parallèlement une autre réforme : la possibilité pour les concubins (de sexe différent ou de même sexe, liés ou non par un PACS), vivant ensemble depuis plus de deux ans, de solliciter une adoption. Une telle initiative présenterait l'avantage de permettre à des couples hétérosexuels ou homosexuels d'adopter un enfant sans que le mariage ne constitue un préalable obligatoire.

103. TGI Paris, 27 juin 2001, Rev. Dr sanit. et soc., 2002, p. 121, note F. Monéger, Rev. trim. Dr civ., 2002, p. 84, obs. J. Hauser, Dr fam., 2001, nº 116, note P. Murat, JCP Ed. G., 2002, I 101, obs. J. Rubellin-Devichi.

104. L'adoption, dans de telles circonstances, n'est pas contraire à la finalité de l'institution, puisqu'il s'agit bien d'établir un lien de filiation. Il ne s'agit nullement d'un détournement d'institution.

105. À condition toutefois que ces enfants aient, « soit dans leur minorité et pendant six ans au moins, soit dans leur minorité et leur majorité et pendant dix ans au moins, reçu de l'adoptant des secours et des soins non interrompus» (art. 786 CGI). 
des situations dramatiques en cas de décès de la mère biologique puisque les enfants ne seraient alors pas séparés de la personne qui les élève depuis toujours. La décision du Tribunal de grande instance présente cependant un inconvénient majeur: la mère biologique est privée de ses droits d'autorité parentale sur ses enfants. C'est en effet l'adoptant qui est seul investi de cette autorité ${ }^{106}$. On peut ici regretter que l'autorité parentale ne puisse être octroyée conjointement à l'adoptant et à l'adopté que dans l'hypothèse de l'adoption de l'enfant du conjoint. La loi du 4 mars 2002, si elle n'a pas remis en cause ce principe ${ }^{107}$, a toutefois ouvert une brèche dans ce système monolithique: elle a assoupli les conditions de la délégation de l'autorité parentale ${ }^{108}$. De surcroît, elle a permis un partage de l'exercice de cette autorité entre délégant et délégataire ${ }^{109}$. Un tel partage entre parent biologique et parent adoptif, ou entre deux parents adoptifs, l'un en vertu d'une adoption plénière, l'autre d'une adoption simple, serait tout à fait indiqué dans le type de situation envisagé. On aurait alors une autre conception de la délégation : celle-ci ne serait plus seulement appelée à jouer dans les situations de crise mais pourrait permettre de reconnaître le rôle, non pas spécifiquement des homosexuels qui élèvent des enfants, mais plus généralement des tiers qui font le choix d'exercer auprès d'eux une véritable fonction parentale ${ }^{110}$.

Il est ainsi tout à fait envisageable que l'homosexualité se voie reconnaître une place au sein des institutions les plus typiquement hétérosexuelles du droit de la famille. On est toutefois en droit de se demander si les homosexuels, lorsqu'ils auront connu les affres du mariage (et inévitablement du divorce) et qu'ils auront été attraits en justice pour se voir imposer le respect des obligations alimentaires liées à la filiation, ne regretteront pas l'époque bénie où ils demeuraient en marge des institutions familiales et ne prôneront pas le retour à l'union libre et au « non droit ».

106. Art. 365 C. civ.

107. Elle a néanmoins permis un exercice commun de l'autorité parentale si l'adoptant et son conjoint en font la déclaration conjointe devant le greffier du tribunal de grande instance (art. 365 C. civ.) alors qu'auparavant le conjoint exerçait seul cette autorité. Dans l'hypothèse d'une ouverture du mariage aux couples de même sexe, cette disposition pourrait avantageusement être transposée pour résoudre les problèmes suscités par de telles situations. Une autre solution serait de permettre un exercice conjoint de l'autorité parentale dans le cadre, plus large, de l'adoption de l'enfant du concubin, ce qui inclurait a fortiori le cas des partenaires liés par un PACS.

108. La délégation est désormais possible quel que soit l'âge du mineur (et non plus seulement si celui-ci a moins de seize ans) et, surtout, elle n'est plus subordonnée à la remise de l'enfant à un tiers (art. 377 C. civ.).

109. Art. 377-1 C. civ.

110. On peut songer plus généralement au concubin de l’un des parents. 